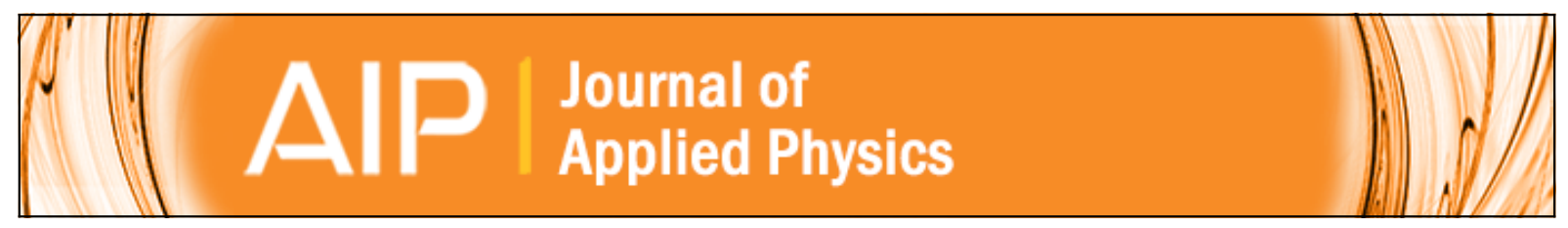

\title{
Adsorption of carbon adatoms to graphene and its nanoribbons
}

C. Ataca, E. Aktürk, H. ahin, and S. Ciraci

Citation: Journal of Applied Physics 109, 013704 (2011); doi: 10.1063/1.3527067

View online: http://dx.doi.org/10.1063/1.3527067

View Table of Contents: http://scitation.aip.org/content/aip/journal/jap/109/1?ver=pdfcov

Published by the AIP Publishing

\section{Articles you may be interested in}

Ferromagnetism, adatom effect, and edge reconstruction induced by Klein boundary in graphene nanoribbons J. Appl. Phys. 113, 194302 (2013); 10.1063/1.4805348

Mechanically tunable magnetism on graphene nanoribbon adsorbed SiO2 surface

J. Appl. Phys. 111, 074317 (2012); 10.1063/1.3702877

Adsorption and diffusion of $3 d$ transition metal atoms on the $\mathrm{GaN}(0001)$ surface

J. Appl. Phys. 110, 083712 (2011); 10.1063/1.3653822

Spin transport properties of single metallocene molecules attached to single-walled carbon nanotubes via nickel adatoms

J. Chem. Phys. 134, 244704 (2011); 10.1063/1.3603446

Mn dimers on graphene nanoribbons: An ab initio study

J. Appl. Phys. 109, 053715 (2011); 10.1063/1.3553849

\section{A|P| $\left.\right|_{\text {Applied Physics }} ^{\text {Journal of }}$}

Journal of Applied Physics is pleased to announce André Anders as its new Editor-in-Chief 


\title{
Adsorption of carbon adatoms to graphene and its nanoribbons
}

\author{
C. Ataca, ${ }^{1,2}$ E. Aktürk, ${ }^{1}$ H. Şahin, ${ }^{1}$ and S. Ciraci ${ }^{1,2, a)}$ \\ ${ }^{1}$ UNAM-Institute of Materials Science and Nanotechnology, Bilkent University, Ankara 06800, Turkey \\ ${ }^{2}$ Department of Physics, Bilkent University, Ankara 06800, Turkey
}

(Received 26 August 2010; accepted 13 November 2010; published online 4 January 2011)

\begin{abstract}
This paper investigates the adsorption of carbon adatoms on graphene and its nanoribbons using first-principles plane wave calculations within density functional theory. The stability at high carbon adatom coverage, migration, and cluster formation of carbon atoms are analyzed. Carbon adatoms give rise to important changes in electronic and magnetic properties even at low coverage. While bare graphene is nonmagnetic semimetal, it is metallized and acquires magnetic moment upon coverage of carbon adatoms. Calculated magnetic moments vary depending on the coverage of adatoms even for large adatom-adatom distances. Electronic and magnetic properties of hydrogen passivated armchair and zigzag nanoribbons show strong dependence on the adsorption site. We also predict a new type of carbon impurity defect in graphene, which has a small formation energy. Interactions between distant carbon adatoms imply a long ranged interaction. () 2011 American Institute of Physics. [doi:10.1063/1.3527067]
\end{abstract}

\section{INTRODUCTION}

Perfect graphene $e^{1,2}$ having two bands linearly crossing at the Fermi level is a nonmagnetic (NM) semimetal. Electron and hole band symmetry leading to ambipolar effect, ${ }^{3}$ carriers showing massless Dirac fermion behavior ${ }^{4}$ near the Fermi level, and high mobility ${ }^{5}$ even at room temperature are only a few properties indigenous to the honeycomb structure of carbon. The properties of graphene are further extended through quasi-one-dimensional (1D) nanoribbon geometries. ${ }^{6}$ In particular, graphene and its nanoribbons attained valuable functionalities through vacancy formation and foreign atom adsorption and substitution. ${ }^{7-11}$ Even if the adsorption of atoms of several elements on graphene have been investigated thoroughly, carbon adatom, which is the constituent element of graphene, and its effects are overlooked. Only recently, carbon adatoms and carbon atomic chains have been observed on graphene using transmission electron microscope (TEM). ${ }^{12}$ Soon after this observation, another TEM study ${ }^{13}$ resolving single carbon adatoms on graphene concluded that bare graphene surfaces are not so clean. These two observations clearly demonstrated that individual carbon adatoms can be adsorbed on graphene surfaces and have stable structure at room temperature. Yet the bonding configuration carbon adatoms and their effects on electronic and magnetic properties remained unexplored.

In this paper, we find that carbon adatoms prefer to adsorb to the bridge sites of graphene and modify the electronic and magnetic properties. The semimetallic bare graphene becomes metallic and attains a net magnetic moment at high carbon coverage. Even more remarkable is that the net magnetic moment shows interesting variation with coverage (or with the distance between two nearest carbon adatoms). Similar effects occur also when carbon adatoms are adsorbed on hydrogen passivated nanoribbons. However, these effects depend on the site where carbon adatom is attached. Notably,

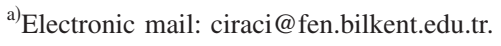

a hydrogen passivated armchair nanoribbon, which is normally NM semiconductor, can change into spin-polarized metal when its surface is covered with carbon adatoms. Whereas antiferromagnetic (AFM) zigzag nanoribbon acquires relatively higher net magnetic moment after carbon adatom adsorption. Our results indicate that carbon adatoms adsorbed on graphene are coupled through $\pi$ - and $\pi^{*}$-states of graphene despite large separations between them. This suggests a long range interaction. While graphene has two equivalent surfaces, the focus of the present paper has been the coverage of one surface by carbon adatoms. Nevertheless, the coverage of both surfaces by carbon adatoms are briefly discussed.

\section{METHOD}

Our predictions are obtained from the state-of-the art spin-polarized, first-principles plane wave calculations ${ }^{14}$ carried out within density functional theory (DFT) using projector augmented-wave (PAW) potentials. ${ }^{15}$ The exchangecorrelation potential is represented by the local density approximation (LDA). Adsorption of carbon adatom is investigated using graphene supercells, which repeat periodically. A plane-wave basis set with kinetic energy cutoff of $900 \mathrm{eV}$ is used. PAW potential with a different core radius is also tested and the same results are obtained. Brillouin zone (BZ) is sampled in the $\mathbf{k}$-space within Monkhorst-Pack scheme, ${ }^{16}$ where the convergence of total energy and magnetic moments with respect to the number of $\mathbf{k}$-points in BZ is carefully tested. All atomic positions and lattice constants are optimized by using the conjugate gradient method, whereby the total energy and atomic forces are minimized. The convergence for energy is chosen as $10^{-5} \mathrm{eV}$ between two consecutive steps, the maximum Hellmann-Feynman forces acting on each atom is less than $0.04 \mathrm{eV} / \AA$ upon ionic relaxation and the pressure is smaller than $1 \mathrm{kbar}$. The stability of carbon covered graphene is examined by calculating frequencies of all phonon modes in BZ within the density 


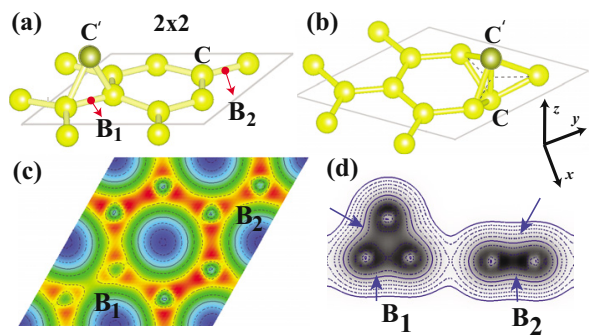

FIG. 1. (Color online) (a) The bridge site bonding geometry of carbon adatom $\left(\mathrm{C}^{\prime}\right)$ adsorbed on the $(2 \times 2)$ supercell of graphene by forming bonds with two $\mathrm{C}$ atoms of the underlying $\mathrm{C}-\mathrm{C}$ bond. $\mathrm{C}-\mathrm{C}^{\prime}-\mathrm{C}$ plane is perpendicular to graphene plane. (b) The adsorption geometry specified as $\mathrm{C} 2$ at the top site corresponds to a local minimum with $0.3 \mathrm{eV}$ higher energy. (c) Charge density contour plots in the lateral $(x, y)$-plane of graphene. (d) Charge density contour plots in a vertical plane (perpendicular to $x$-axis) passing through $\mathrm{C}-\mathrm{C}^{\prime}-\mathrm{C}$ and a regular $\mathrm{C}-\mathrm{C}$ bond of graphene. $\mathrm{B}_{1}$ is the bridge site where $\mathrm{C}^{\prime}$ is attached; $\mathrm{B}_{2}$ is a bridge site without $\mathrm{C}^{\prime}$. Weakening of $\mathrm{C}-\mathrm{C}$ bonds beneath $\mathrm{C}^{\prime}$ is easily recognized. Contour spacings are 0.001 electrons. $z$-axis is perpendicular to graphene plane.

functional perturbation theory using PWSCF package. ${ }^{17}$ All calculations are performed at $\mathrm{T}=0 \mathrm{~K}$. For the sake of confirmation, local magnetic moments are also calculated using SIESTA code ${ }^{18}$ within LDA. In these calculations, TroullierMartin type norm-conserving pseudopotential are used and the valance electrons are described by localized pseudoatomic orbitals with a double zeta singly polarized basis set. Energy cut off is taken as 500 Ry.

\section{ADSORPTION ON TWO-DIMENSIONAL (2D) GRAPHENE}

Different levels of one-sided periodic coverage of carbon adatom (labeled as $C^{\prime}$ in the rest of the paper) are considered. This is achieved by attaching one $\mathrm{C}^{\prime}$ to each $(n$ $\times n$ ) supercell of graphene for $n=1,2,3, \ldots, 9$, which lead to periodic adatom coverage specified as $\Theta=1 / 2 n^{2}$. The favorable adsorption site is determined by placing the adatom in different possible adsorption sites above graphene, such as the top ( $\mathrm{T}$, top of carbon atoms), the bridge (B, above the $\mathrm{C}-\mathrm{C}$ bond of graphene), the hollow ( $\mathrm{H}$, above the center of hexagons) sites, and each time by optimizing structures to obtain minimum energy and atomic forces. The binding energy for the bridge site, in Fig. 1(a) is found to be maximum for all $(n \times n)$ supercells. Carbon atom placed to the top or hollow site moves to the bridge site upon relaxation. For example, the energy is lowered by $0.86 \mathrm{eV}$ by going from the top site to the bridge site. The binding energies are obtained from the expression, $E_{b}=E_{G r}+E_{C}-E_{T}$. Here $E_{T}$ and $E_{G r}$ are the total energies of graphene $+\mathrm{C}^{\prime}$ and bare graphene per $(n \times n)$ supercell. $E_{C}$ is the total energy of single $C$ atom calculated in the same supercell with and without spin polarization. The spin polarized calculations yield $2 \mu_{\mathrm{B}}$ magnetic moment on a free carbon atom, which is $\sim 1.12 \mathrm{eV}$ more favorable energetically. ${ }^{19}$ Relevant structural parameters, binding energies, and magnetic moments calculated for various periodic coverage of $\mathrm{C}^{\prime}$ are given in Table $\mathrm{I}$.

We note that the $\mathrm{C}-\mathrm{C}$ bond below $\mathrm{C}^{\prime}$ elongates and its charge is slightly delocalized [as shown in Fig. 1(c) and 1(d)]. We also note a specific adsorption geometry C2 at the top site [as described in Fig. 1(b)], where one carbon atom normally located at the corner of the hexagon is replaced by two carbon atoms one of them being above the graphene plane, the other below. This configuration corresponds to a local minimum, which has $0.3 \mathrm{eV}$ smaller binding energy than the energy of the bridge site and has spin-unpolarized, metallic ground state for $\Theta=1 / 8$. Owing to its small formation energy, C2 can be a candidate for a carbon impurity defect.

\section{A. Stability and migration of carbon adatoms}

The highest coverage we studied is $\Theta=1 / 2$, which corresponds to one $\mathrm{C}^{\prime}$ adsorbed to each $(1 \times 1)$ cell. However, this coverage cannot remain uniform but forms clusters upon structure optimization. Nonetheless, the periodic coverages of $\mathrm{C}^{\prime}$ with $\Theta \leq 1 / 8$ and hence the adatom-adatom distance $l_{\mathrm{C}^{\prime}-\mathrm{C}^{\prime}} \geq 4.94 \AA$ are stable and do not form clusters at $\mathrm{T}$ $=0 \mathrm{~K}$. For the coverage $\Theta=1 / 8$, the ground state is found to be $\mathrm{NM}$ and has a binding energy, $E_{b}=2.43 \mathrm{eV}$ (calculated relative to the energy of a free carbon atom in magnetic ground state.) In view of the calculated binding energies of other atoms ${ }^{7-11}$ carbon forms stronger bonds with graphene surface.

The phonon dispersion curves of the coverage $\Theta=1 / 8$, which has the smallest $C^{\prime}-C^{\prime}$ distance of $4.64 \AA$ among other periodic $\mathrm{C}^{\prime}$ coverages are studied in this work. In Fig. 2 the phonon branches along relevant symmetry directions and corresponding total density of states of bare and $\Theta$

TABLE I. Calculated values for optimized structure of the single carbon adatom $\left(\mathrm{C}^{\prime}\right)$ adsorbed to the bridge site of each $(n \times n)$ supercell of graphene where $n=2,3, \ldots, 9$. Average $\mathrm{C}^{\prime}-\mathrm{C}$ distance, $d_{\mathrm{C}^{\prime}-\mathrm{C}}$; the length of a regular $\mathrm{C}-\mathrm{C}$ bond in graphene, $d_{\mathrm{C}-\mathrm{C}}$; the length of the $\mathrm{C}-\mathrm{C}$ bond below the adatom $\mathrm{C}^{\prime}, d_{\mathrm{C}-\mathrm{C}}^{\prime}$; the lattice constant, $a$; the binding energy, $E_{b}$ (the values in the parenthesis are calculated relative to the free atom energies of $\mathrm{C}$ in NM state); magnetic moment per supercell, $\mu$; and spin-polarization at the Fermi energy, $P\left(E_{F}\right)$. NM refers to NM state.

\begin{tabular}{cccccccc}
\hline \hline$\Theta\left(1 / 2 n^{2}\right)$ & Supercell $(n \times n)$ & $\begin{array}{c}d_{\mathrm{C}^{\prime}-\mathrm{C}} \\
(\AA)\end{array}$ & $\begin{array}{c}d_{\mathrm{C}-\mathrm{C}} \\
(\AA)\end{array}$ & $\begin{array}{c}d_{\mathrm{C}-\mathrm{C}}^{\prime} \\
(\AA)\end{array}$ & $\begin{array}{c}a \\
(\AA)\end{array}$ & $\begin{array}{c}E_{b} \\
(\mathrm{eV})\end{array}$ & $\begin{array}{c}\mu \\
\left(\mu_{\mathrm{B}}\right)\end{array}$ \\
\hline $1 / 8$ & $2 \times 2$ & 1.54 & 1.43 & 1.49 & 4.94 & $2.43(3.55)$ & $\mathrm{NM}$ \\
$\left(\mu_{\mathrm{B}}\right)$
\end{tabular}


(a)

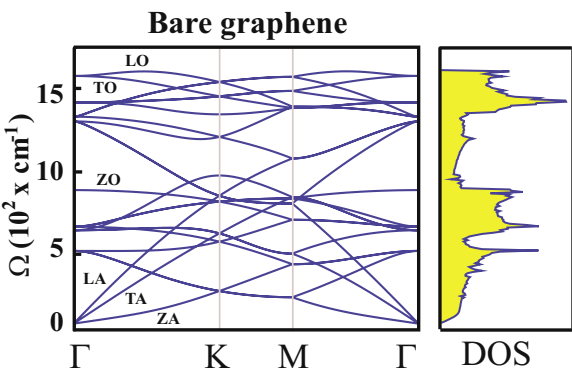

(c)
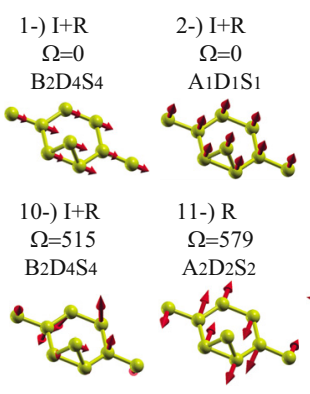

19-) R

$\Omega=1285$

$\mathrm{A} 2 \mathrm{D} 2 \mathrm{~S} 2$

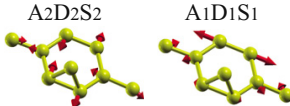

3-) I+R

$\Omega=0$

$\mathrm{B}_{1} \mathrm{D}_{3} \mathrm{~S}_{3}$

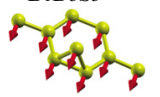

12-) I+R

$\Omega=594$

B1D3S3

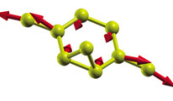

21-) I+R

$\Omega=1330$

$\mathrm{B} 1 \mathrm{D}_{3} \mathrm{~S} 3$

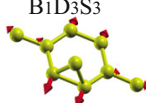

4-) I+R

$\Omega=241$

$\mathrm{B} 1 \mathrm{D} 3 \mathrm{~S} 3$

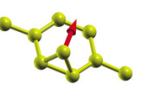

13-) $\mathrm{I}+\mathrm{R}$

$\Omega=620$

$\mathrm{B} 2 \mathrm{D} 4 \mathrm{~S} 4$

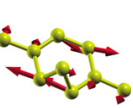

22-) I+R

$\Omega=1366$

$\mathrm{B} 2 \mathrm{D} 4 \mathrm{~S} 4$

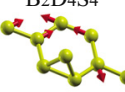

(b)
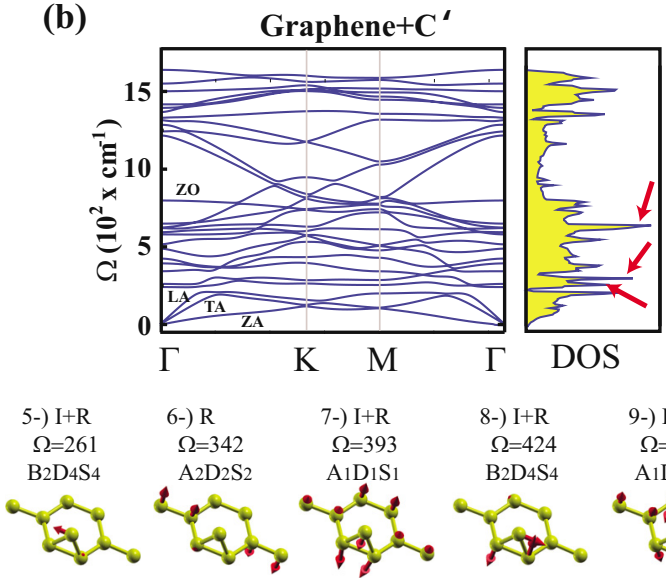

14-) I+R

$\Omega=623$

A1D1S1

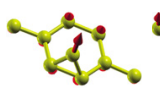

23-) I+R

$\Omega=1394$

A1DiS

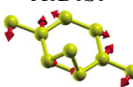

sects
6-) $\mathrm{R}$ 7-) $\mathrm{I}+\mathrm{R}$

$\Omega=342$

$\mathrm{A} 2 \mathrm{D} 2 \mathrm{~S} 2$

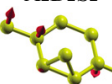

$\mathrm{A} 1 \mathrm{D} 1 \mathrm{~S} 1$

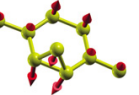

15-) I+R

$\Omega=649$

16-) I+R

$\Omega=798$

$\mathrm{B} 2 \mathrm{D} 4 \mathrm{~S} 4$

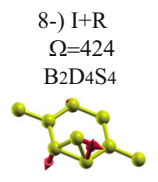

17-) I+R

$\Omega=1216$

$\Omega=1216$
A1D1S1

9-) I+R

$\Omega=488$

A1DiS

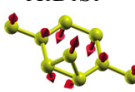

18-) I+R

$\Omega=1242$

$\mathrm{B} 2 \mathrm{D} 4 \mathrm{~S} 4$

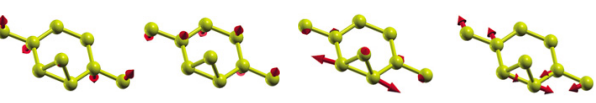

24-) $\mathrm{R} \quad 25$-) $\mathrm{I}+\mathrm{R}$

$\Omega=1416$

$\mathrm{A} 2 \mathrm{D} 2 \mathrm{~S} 2$

$\Omega=1501$

$\mathrm{B}_{1} \mathrm{D}_{3} \mathrm{~S}_{3}$

26-) I+R

$\Omega=1550$

27-) R

$\Omega=1639$

$\mathrm{A} 2 \mathrm{D} 2 \mathrm{~S} 2$
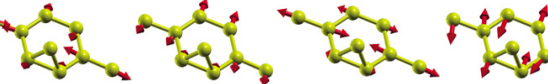

FIG. 2. (Color online) Phonon frequencies, $\Omega(\mathrm{k})$ and corresponding density of states calculated along symmetry direction of the BZ of the $(2 \times 2)$ supercell. (a) Bare graphene. (b) Graphene $+C^{\prime}$ corresponding to $\Theta=1 / 8$. Major contributions of $C^{\prime}$ is indicated by arrows in DOS. (c) Description of Raman (R) and infrared (I) active modes of graphene $+\mathrm{C}^{\prime}$ and their frequencies $\Omega(\mathrm{k})$ in per centimeter.

$=1 / 8 \mathrm{C}^{\prime}$ covered graphene are calculated for the $(2 \times 2) \mathrm{su}-$ percell. The stability at $\Theta=1 / 8$ is assured, since all phonon modes in BZ have positive frequencies. However, the density of states are modified through the adsorption of $\mathrm{C}^{\prime}$. The peaks in the total density of states associated with specific modes of adatoms are indicated in Fig. 2(b).

In Fig. 2(a) six modes of bare graphene become doubly degenerate. The out-of-plane acoustic mode ZA has quadratic dispersion, which is characteristic of structures having monolayer honeycomb structures. ${ }^{20}$ Another optical mode, $\mathrm{ZO}$ at $900 \mathrm{~cm}^{-1}$, which is dispersionless near $\Gamma$ point, is related with the out-of-plane motion of atoms. Highest two optical modes LO and TO (with $1586 \mathrm{~cm}^{-1}$ ) are degenerate at $\Gamma$ point. Detailed analysis of $\mathrm{LO}$ and TO modes regarding their vibrational properties and couplings to electronic states leading to Kohn anomaly were also reported. ${ }^{21,22}$ Upon the coverage of $\Theta=1 / 8$ of $C^{\prime}$ the $D_{6 h}$ symmetry of graphene turns into the $\mathrm{C}_{2 v}$ (or $\mathrm{mm} 2$ ) point group. It is seen from Fig. 2(b) that all the degenerate modes are separated due to adsorbed $\mathrm{C}^{\prime}$. Upon adatom adsorption, a narrow gap opens between acoustic and optical modes. In Fig. 2(c), Raman (R) and infrared (I) active modes together with their frequencies at $\mathbf{k}=0$ are shown. While first and third acoustic modes related with in-plane propagation, second mode corresponds to the out-of-plane motion of all $\mathrm{C}$ atoms in the unitcell. Out of three acoustic modes, fourth, fifth, and 14th modes in Fig. 2(c) are driven from the carbon adatom and the remaining originate from graphene.

Apart from the stability of $\mathrm{C}^{\prime}$ covered graphene, the migration of $\mathrm{C}^{\prime}$ itself on graphene surface is of crucial importance. The migration of a single $\mathrm{C}^{\prime}$ atom is hindered by an energy barrier of $\sim 0.30 \mathrm{eV}$ at $\mathrm{T}=0 \mathrm{~K}$. This barrier is, however, lowered at the proximity of a second $\mathrm{C}^{\prime}$ at a distance, $l_{\mathrm{C}^{\prime}-\mathrm{C}^{\prime}} \leq 3.40 \AA$. However, the diffusion of $\mathrm{C}^{\prime}$ at room temperature can be significant. Consequently, $\mathrm{C}^{\prime}$ atoms migrate readily to form clusters. In fact, the diffusion of carbon adatom as well as clusters formed on the surface of graphene have been demonstrated by TEM images taken at room temperature. ${ }^{12}$

Before we close this section, we discuss the case where both surfaces of graphene is covered. Here we distinguish various alternatives, such as (i) each surface is covered by different elements; (ii) each surface is covered by $\mathrm{C}^{\prime}$ but at different values of coverage, $\Theta$; (iii) each surface is covered by $\mathrm{C}^{\prime}$ at the same coverage. Each alternative results in different electronic structure, magnetic moment, work function, etc. This way both surfaces are utilized for specific functionalities. We treated only the double-sided coverage, where two $\mathrm{C}^{\prime}$ 's are adsorbed to the bridge sites in the same (2 $\times 2)$ supercell but at different sides. Here the crucial parameter is the distance between top and bottom $\mathrm{C}^{\prime}$, i.e., $d_{\mathrm{C}^{\prime}-\mathrm{C}^{\prime}}$. The calculated binding energies are $3.16 \mathrm{eV}(3.89 \mathrm{eV})$ and $2.94 \mathrm{eV}(3.45 \mathrm{eV})$ for $d_{\mathrm{C}^{\prime}-\mathrm{C}^{\prime}}=3.92 \AA$ and $3.64 \AA$, respectively. Here, the first entry is the average binding energy, the one in the parenthesis is the binding energy of the second $\mathrm{C}^{\prime}$. It appears that the binding energy is $\sim 2.4 \mathrm{eV}$ for very large $d_{\mathrm{C}^{\prime}-\mathrm{C}^{\prime}}$. However, as $d_{\mathrm{C}^{\prime}-\mathrm{C}^{\prime}}$ decreases to a critical distance, the binding energy increases passing through a maximum value. When a carbon adatom is placed at a bridge site just below the existing $\mathrm{C}^{\prime}$ or to the next nearest neighbor bridge site below, a local reconstruction takes place, which results in the formation of a $\mathrm{C} 2$ defect. 

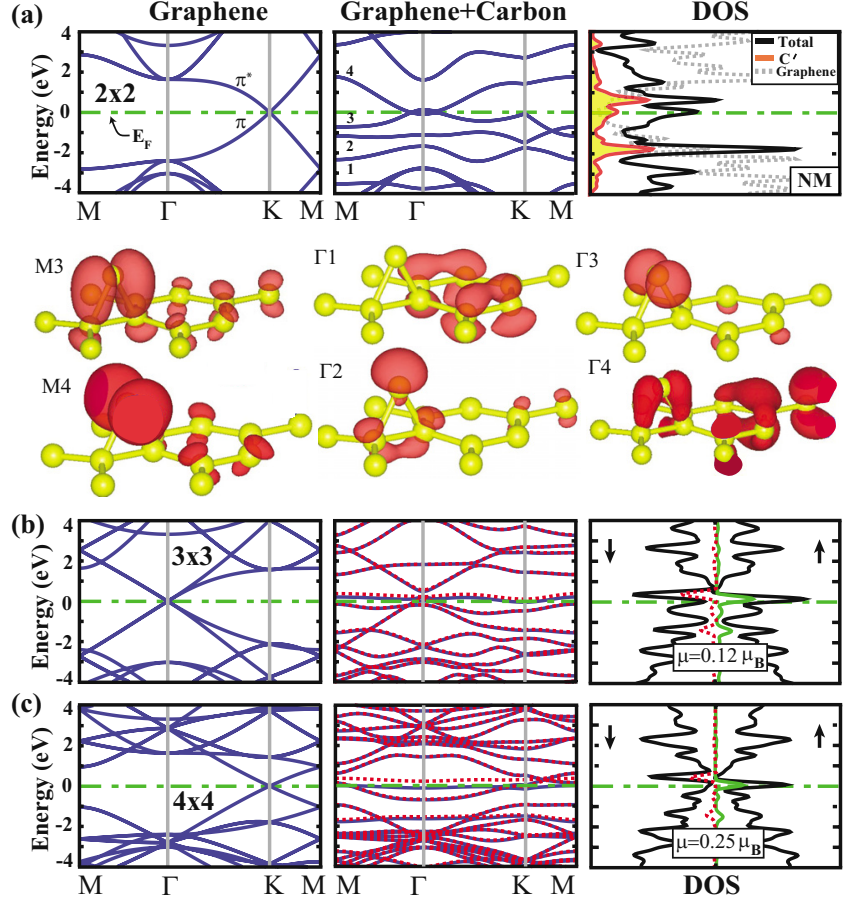

FIG. 3. (Color online) (a) Electronic energy band structure of one $\mathrm{C}^{\prime}$ adsorbed to each $(2 \times 2)$ supercell of graphene with the corresponding total and adatom projected density of states, which is scaled by two for the sake of clarity. The ground state is NM. The total density of states of bare graphene is included with dashed light (gray) lines for comparison. Isosurfaces of selected states are also shown. (b) and (c) same for one $\mathrm{C}^{\prime}$ adsorbed to each $(n \times n)$ supercell of graphene with total and adatom projected density of states of spin-up and spin-down states for $n=3$ and 4, respectively. Band structures of bare graphene folded to BZ of $(n \times n)$ supercell is presented in the left panels. Adatom $\mathrm{C}^{\prime}$ is adsorbed to the bridge sites. Zero of energy is set to the Fermi level shown by dashed dotted light (green) lines. Spin-up and spin-down states projected to the adatom are shown by light (green) and dotted (red) lines, respectively. The magnetic moments, $\mu$ (in Bohr magneton) calculated for each $(n \times n)$ supercell are also indicated. Continuous dark (blue) bands indicate spin-up states; dotted (red) lines are spin-down states.

\section{B. Electronic structure}

Even if a periodic coverage of $\mathrm{C}^{\prime}$ is only a model to use in the band theory, recent advances in nanotechnology has made the fabrication of periodic nanomeshes possible. ${ }^{23}$ Here we consider the electronic band structure and spin projected total density of states for different $\Theta=1 / 2 n^{2}$. We calculate energy bands for $n=2-9$ and present in Fig. 3 only for $n=2-4$. Here the analysis of new states appearing near the Fermi level, $E_{F}$, is essential for better understanding of the electronic structure. The charge density analysis of these states indicates that the orbitals of $\mathrm{C}^{\prime}$ (namely, one $s p^{2}$-like orbitals perpendicular to the plane of graphene, other two $s p^{2}$-like orbitals forming two $\mathrm{C}^{\prime}-\mathrm{C}$ bonds and one $p_{x}$-orbitals perpendicular to the $\mathrm{C}-\mathrm{C}^{\prime}-\mathrm{C}$ plane) are combined with $\pi$ - and $\pi^{*}$-orbitals of bare graphene and form new bands near the Fermi level shown in Fig. 3(a). The flat band driven from perpendicular $s p^{2}$ orbitals is filled and gives rise to a peak at $\sim 2 \mathrm{eV}$ below $E_{F}$ in the total density of states. Bands generated from the hybridization of other $s p^{2}$-orbitals of $\mathrm{C}^{\prime}$ with the $\pi$ - and $\pi^{*}$-states of graphene occur near $E_{F}$ and attribute a metallic character. The band above $E_{F}$ is formed from antibonding combination of $p$-orbital of $\mathrm{C}^{\prime}$ perpendicular to $\mathrm{C}-\mathrm{C}^{\prime}-\mathrm{C}$ plane and $p^{*}$-orbitals of graphene. The effect of $\mathrm{C}^{\prime}$ on the electronic structure can be deduced by comparing the bands of bare graphene folded to the $(n \times n)$ BZ and its density of states presented in the same figure.

Our calculations predict that the ground state of $(n \times n)$ graphene $+C^{\prime}$ with $n>2$ is spin-polarized due to broken spin degeneracy of bands near $E_{F}$. For example, while the $\Theta$ $=1 / 8$ coverage is NM (or spin-unpolarized) ground state with a minute energy difference from the spin-polarized excited state, $\Theta=1 / 18$ coverage with relatively larger $C^{\prime}-C^{\prime}$ distance of $7.37 \AA$ has a significant magnetic moment $\mu$ $=0.12 \mu_{\mathrm{B}}$. The coverage of $\Theta=1 / 32$ has even larger $\mathrm{C}^{\prime}-\mathrm{C}^{\prime}$ distance, namely, $l_{\mathrm{C}^{\prime}-\mathrm{C}^{\prime}}=9.80 \AA$ and has metallic state. Its spin-polarized ground state is found to be energetically favorable by $12 \mathrm{meV}$, and its net magnetic moment is $0.25 \mu_{\mathrm{B}}$ per $(4 \times 4)$ supercell.

As the supercell size $(n \times n)$ increases and hence $\Theta$ decreases further, the ground state of the graphene $+\mathrm{C}^{\prime}$ system remains metallic and spin-polarized. However, despite increasing value of $l_{\mathrm{C}^{\prime}-\mathrm{C}^{\prime}}$ the value of the magnetic moment continues to vary. For example, while the value of $\mu$ $=0.27 \mu_{\mathrm{B}}$ for $n=5$, it decreases to $\mu=0.20 \mu_{\mathrm{B}}$ for $n=6$. Only from $\Theta=1 / 98$ on corresponding to $(7 \times 7)$ and hence $l_{\mathrm{C}^{\prime}-\mathrm{C}^{\prime}}=17.13 \AA$ the magnetic moment converges to a value of $\mu=\sim 0.25 \mu_{\mathrm{B}}$. These trends and values of magnetic moments are also tested by performing calculations using generalized gradient approximation (GGA) for the exchangecorrelation potential and also using local basis set with SIESTA code. ${ }^{18}$ Our conclusions are confirmed by these calculations.

In addition to magnetic states, the spin-polarization at the Fermi level, $P=\left[D\left(E_{F}, \uparrow\right)-D\left(E_{F}, \downarrow\right)\right] /\left[D\left(E_{F}, \uparrow\right)\right.$ $\left.+D\left(E_{F}, \downarrow\right)\right]$ (calculated in terms of the density of states spin-up (spin-down) states at $E_{F}, D\left(E_{F}, \uparrow\right)$, and $\left(D\left(E_{F}, \downarrow\right)\right)$ exhibit substantial variation with $n$ (see Table I). We note that small variation in $P$ for $\Theta \leq 1 / 98$ is due to change in the size of the supercell.

Clearly, these variations in spintronic and magnetic properties despite $l_{\mathrm{C}^{\prime}-\mathrm{C}^{\prime}}$ as large as $17.13 \AA$ indicate a rather long ranged couplings between carbon adatoms through $\pi$ and $\pi^{*}$-states of graphene. Not only magnetic properties but also the bands near $E_{F}$ in Fig. 3 display similar long ranged effects. For $n=2$, the mixing of the orbitals of $\mathrm{C}^{\prime}$ and graphene $\pi$-orbitals is significant. Notably, the bands around $E_{F}$ are altered and a wide gap is opened between linearly crossing bands at the Dirac points. Because of band folding, the bands are crossed at $\Gamma$ point instead of $K$ point for specific $n$. Up to $n=7$ the bands of bare graphene continue to be affected by carbon adatoms, which is visualized by the dispersion of bands and the opening a gap where the bands of bare graphene are linearly crossed.

Present results pertaining the adsorption site and diffusion barrier are in fair agreement with earlier first-principles plane wave studies using GGA. ${ }^{24-26}$ They predicted that carbon atoms adsorbed to $(n \times n)$ supercell for $n=5,6$ and 7 generates a magnetic moment of $\sim 0.5 \mu_{\mathrm{B}}$. However, our study for $n=2-9$ demonstrates that the magnetic moment as well as $P$ vary with $n$ up to $n=9$. We also investigated the 
effects of carbon adatoms on the electronic structure and show that the adatom driven metallic band exhibits a significant width. Additionally, present study focuses to a fundamental issue and reveals that the coupling between carbon adatoms on graphene can exists for distances as long as $17.13 \AA$. It is known that the Friedel oscillations ${ }^{27}$ in the free electron gas systems decay as $\sim 1 / l^{D}, D$ being the dimensionality of the system. Here, the states of $\pi$ - and $\pi^{*}$-bands of graphene display a free electron like behavior, since the charge density having a node at the graphene plane is not affected by the ion cores. More recently, the free electron behavior and resulting Friedel oscillation decaying with $1 / l$ are clarified for 1D carbon atomic chains. ${ }^{28}$

\section{ADSORPTION ON GRAPHENE NANORIBBONS}

Interesting effects of $\mathrm{C}^{\prime}$ have been also revealed for hydrogen saturated graphene nanoribbons. Owing to the breaking of $\mathrm{C}_{6}$ rotation symmetry in nanoribbons, the differences between various bridge sites of a nanoribbon are distinguished. Moreover, the effect of $\mathrm{C}^{\prime}$ changes whether it is adsorbed at the edge or at the center of the ribbon. In Fig. 4 we show the electronic and magnetic properties of bare hydrogen passivated armchair nanoribbon (i.e., adatom free), as well as that with carbon adatoms. Here one $\mathrm{C}^{\prime}$ adsorbed to each supercell consisting of double primitive unit cell, (2 $\times 1$ ). Hydrogen passivated bare armchair nanoribbon with $N=12$ (which is the number of $\mathrm{C}-\mathrm{C}$ dimers in the primitive unit cell parallel to the axis of the ribbon) is normally a $\mathrm{NM}$ semiconductor. It remains to be $\mathrm{NM}$ semiconductor but a smaller band gap is achieved, when $\mathrm{C}^{\prime}$ is adsorbed to either A- or E-site. However, the same nanoribbon can change to a spin-polarized metal with $\mu=0.35 \mu_{\mathrm{B}}$ upon the adsorption of carbon adatom at the B-site, since spin degeneracy is broken and the energy of the spin-down band is lowered to overlap with the valance band. While the NM state is only an excited state $20 \mathrm{meV}$ above, the metallicity can be questioned, since the band gap is usually underestimated by DFT. The binding energies depend also on the adsorption site; and they are $2.33 \mathrm{eV}, 2.32 \mathrm{eV}$, and $3.81 \mathrm{eV}$ for A-, B-, and E-sites, respectively. The energy difference between A- and $\mathrm{B}$-sites is only $9 \mathrm{meV}$ and hence within the accuracy limits of DFT. E-site is $1.48 \mathrm{eV}$ energetically more favorable relative to A-site, since its binding configuration differs from the bridge site. Similar trends persist even if $\mathrm{C}^{\prime}-\mathrm{C}^{\prime}$ distance increases through adsorption to $(3 \times 1)$ and $(4 \times 1)$ supercells, except that the energy difference between A- and B-sites increases. Similar to 2D graphene, the magnetic moment of B-site varies as the size of the supercell increases from $n=(2 \times 1)$ to $n=(4 \times 1)$.

Next we considered a hydrogen passivated zigzag nanoribbon having $N=6$ (which is the number of zigzag chains along the ribbon axis) or the width $w=13.41 \AA$ and performed calculation for single $C^{\prime}$ adsorbed to each $n=(4$ $\times 1)$ supercell. While hydrogen saturated bare zigzag nanoribbons are semiconducting and have ferromagnetically ordered spins at the same edge but antiferromagnetically coupled with the opposite edge, it becomes spin-polarized metal when $\mathrm{C}^{\prime}$ is adsorbed at A- and B-sites with magnetic

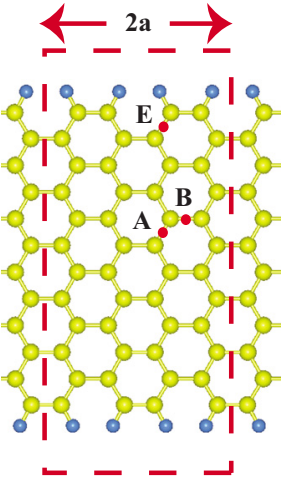

(a)

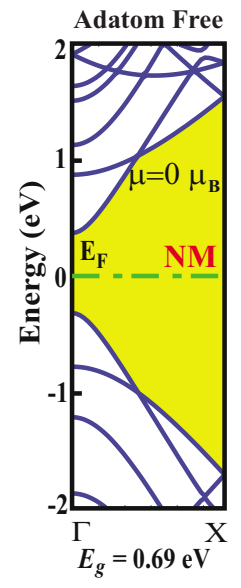

(b)

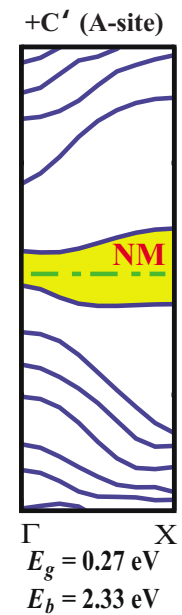

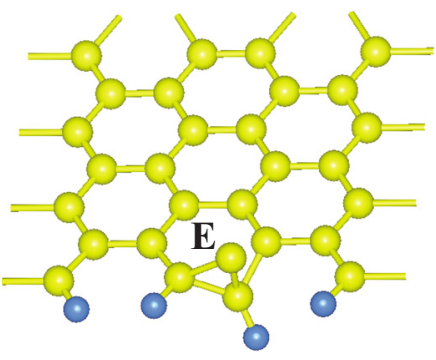

(c)

(d)

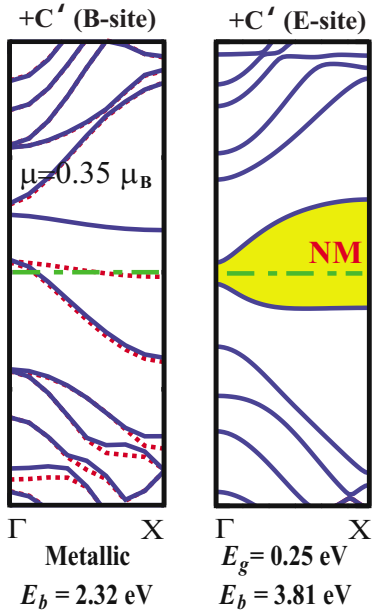

FIG. 4. (Color online) Effect of carbon adatom $\mathrm{C}^{\prime}$ on the electronic and magnetic properties of hydrogen passivated armchair graphene nanoribbons are calculated in a $(2 \times 1)$ supercell comprising double primitive unit cell with $2 a=8.50 \AA$. The width of the nanoribbons is $N=12$ or $w=$ $\sim 15.35 \AA$. The supercell is delineated by dashed lines and the final atomic structure corresponding to carbon adatom adsorbed at the E-site forming a $\mathrm{C} 2$ bonding is shown above. (a) Bare hydrogen saturated armchair nanoribbon and its band structure folded to $(2 \times 1)$ supercell. [(b)-(d)] Same nanoribbon with $\mathrm{C}^{\prime}$ is adsorbed to the sites, $\mathrm{A}, \mathrm{B}$, and $\mathrm{E}$. The zero of energy is set to the Fermi level. In (c), the solid (blue) and dashed (red) lines are spin-up and spin-down bands, respectively. $E_{g}, E_{b}$, and $\mathrm{NM}$, respectively, stand for the band gap, the binding energy and the NM state.

moments $\mu=2 \mu_{\mathrm{B}}$ and $1.47 \mu_{\mathrm{B}}$ per supercell, respectively. However, the nanoribbon becomes spin-polarized semiconductor with $\mu=2 \mu_{\mathrm{B}}$ per supercell when $\mathrm{C}^{\prime}$ is adsorbed to E-site. Interestingly, the binding configuration of $\mathrm{C}^{\prime}$ at E-site turns out to be C2-like. The binding energies of A-, B-, and E-sites are, respectively, $2.42 \mathrm{eV}, 2.70 \mathrm{eV}$, and $4.86 \mathrm{eV}$. Clearly, for both armchair and zigzag nanoribbons the edge site are favored energetically (Fig. 5).

It should be noted that the coupling with both magnetic edge states of the nanoribbon underlie the high magnetic moment achieved as a result of the adsorption of carbon adatom. In fact, any edge of the nanoribbon, which have normally ferromagnetic order, turns out to be $\mathrm{NM}$ when a $\mathrm{C}^{\prime}$ approaches to it. Notably, for A-site there are excited states with relatively smaller total magnetic moment just above the ground state. Nonetheless, the local magnetic moment at carbon adatom appears to be unaltered and has a value comparable to that of $\mathrm{C}^{\prime}$ adsorbed to $2 \mathrm{D}$ graphene. It is expected that the coupling with magnetic edge states becomes negli- 


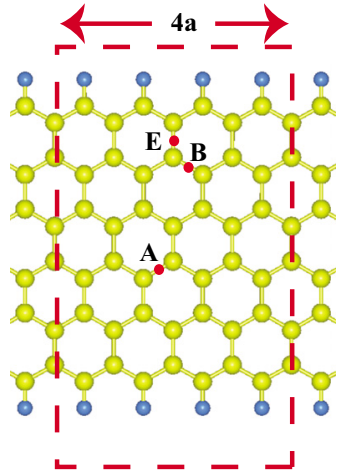

(a)

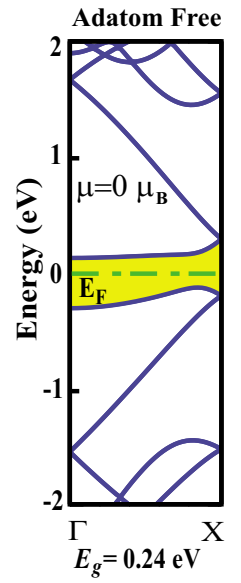

(b)

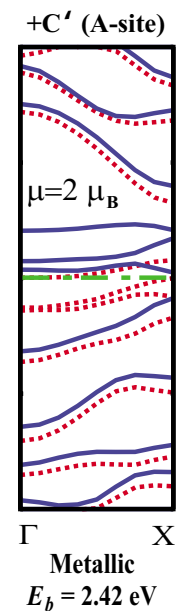

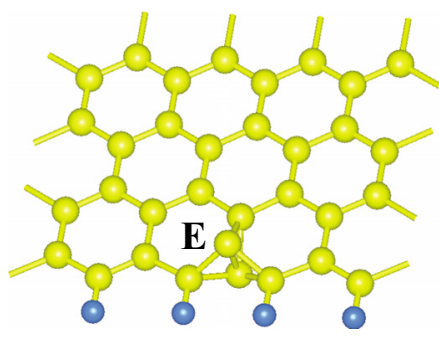

(c)

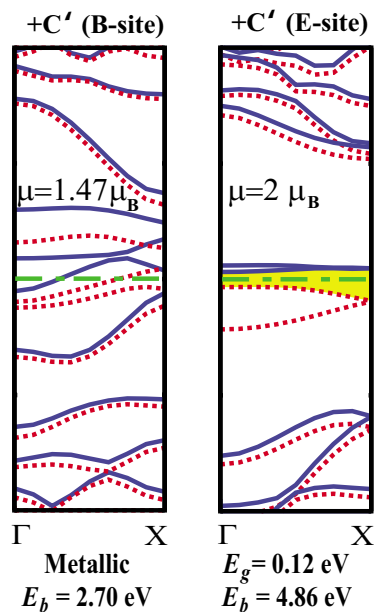

FIG. 5. (Color online) Effect of carbon adatom $\mathrm{C}^{\prime}$ on the electronic and magnetic properties of hydrogen saturated zigzag graphene nanoribbons are calculated in a $(4 \times 1)$ supercell comprising four primitive unit cell with $4 a=9.74 \AA$. The supercell is delineated by dashed lines and atomic structure corresponding to carbon adatom adsorbed at the E-site forming a C2like bonding is shown above. The width of the zigzag nanoribbon $N=6$ is $w=13.41 \AA$. (a) Bare, hydrogen saturated zigzag nanoribbon and its band structure folded to $(4 \times 1)$ supercell. The ribbon has the AFM ground state. [(b)-(d)] Same nanoribbon with $\mathrm{C}^{\prime}$ in different locations A, B, and E. The solid (blue) and dotted (red) lines are spin-up and spin-down bands, respectively. $E_{g}$ and $E_{b}$ stand for the band gap and the binding energy, respectively. The zero of energy is set to the Fermi level and indicated by dashed dotted (green) lines..

gible for very wide zigzag nanoribbons and the magnetic moment at adatom recovers the value of $2 \mathrm{D}$ graphene.

\section{CONCLUSION}

In summary, we studied the periodic adsorption of carbon adatom to graphene in a wide range of coverage, $1 / 162 \leq \Theta \leq 1 / 2$. The electronic and magnetic properties are influenced after carbon adatom coverage. A semimetallic graphene changes to be metallic and spin-polarized upon the periodic adsorption of carbon adatoms. The variation in magnetic moments for the adatom-adatom distance as large as $\sim 17.13 \AA$ indicates a long ranged interaction between adatoms. The adsorption of carbon adatoms on the hydrogen saturated armchair and zigzag nanoribbons also result in major modifications of electronic and magnetic properties. Hydrogen passivated bare armchair nanoribbon, which is a NM semiconductor, becomes either narrow band gap semiconductor or a spin-polarized metal depending on the adsorption site of carbon. Similarly, hydrogen passivated bare zigzag nanoribbon, which is an AFM semiconductor, becomes either spin-polarized metal or semiconductor depending on the adsorption site of carbon adatom. Present results suggest that a single, isolated carbon atom adsorbed to graphene has local impurity states near the Fermi level and a local magnetic moment of $\sim 0.25 \mu_{\mathrm{B}}$. Finally, we note that graphene acquires new functionalities through chemically active carbon adatoms on its surfaces.

\section{ACKNOWLEDGMENTS}

S.C. and H.Ş. acknowledge financial support by the Scientific and Technological Research Council of Turkey, TÜBITAK, under the Project No. 108T234. Authors also thank D. Alfe for fruitful discussions.

${ }^{1}$ Y. Zhang, Y.-W. Tan, H. L. Stormer, and P. Kim, Nature (London) 438, 201 (2005)

${ }^{2}$ C. Berger, Z. Song, X. Li, X. Wu, N. Brown, C. Naud, D. Mayou, T. Li, J. Hass, A. N. Marchenkov, E. H. Conrad, P. N. First, and W. A. de Heer, Science 312, 1191 (2006).

${ }^{3}$ A. K. Geim and K. S. Novoselov, Nature Mater. 6, 183 (2007).

${ }^{4}$ K. S. Novoselov, A. K. Geim, S. V. Morozov, D. Jiang, M. I. Katsnelson, I. V. Grigorieva, S. V. Dubonos, and A. A. Firsov, Nature (London) 438, 197 (2005)

${ }^{5}$ K. S. Novoselov, A. K. Geim, S. V. Morozov, D. Jiang, Y. Zhang, S. V. Dubonos, I. V. Grigorieva, and A. A. Firsov, Science 306, 666 (2004).

${ }^{6}$ X. Li, X. Wang, L. Zhang, S. Lee, and H. Dai, Science 319, 1229 (2008). ${ }^{7}$ H. Sevinçli, M. Topsakal, E. Durgun, and S. Ciraci, Phys. Rev. B 77, 195434 (2008).

${ }^{8}$ K. T. Chan, J. B. Neaton, and M. L. Cohen, Phys. Rev. B 77, 235430 (2008).

${ }^{9}$ C. Ataca, E. Aktürk, S. Ciraci, and H. Ustunel, Appl. Phys. Lett. 93, 043123 (2008).

${ }^{10}$ C. Ataca, E. Aktürk, and S. Ciraci, Phys. Rev. B 79, 041406(R) (2009).

${ }^{11}$ E. Aktürk, C. Ataca, and S. Ciraci, Appl. Phys. Lett. 96, 123112 (2010).

${ }^{12}$ J. C. Meyer, C. O. Girit, M. F. Crommie, and A. Zettl, Nature (London) 454, 319 (2008).

${ }^{13}$ U. Bangert, M. H. Gass, A. L. Bleloch, R. R. Nair, and J. Eccles, Phys. Status Solidi A 206, 2115 (2009).

${ }^{14}$ G. Kresse and J. Furthmuller, Phys. Rev. B 54, 11169 (1996).

${ }^{15}$ P. E. Blöchl, Phys. Rev. B 50, 17953 (1994).

${ }^{16}$ H. J. Monkhorst and J. D. Pack, Phys. Rev. B 13, 5188 (1976).

${ }^{17} \mathrm{~S}$. Baroni, A. Del Corso, S. Girancoli, and P. Giannozzi, http:/ www.pwscf.org/

${ }^{18}$ P. Ordejón, E. Artacho and J. M. Soler, Phys. Rev. B 53, 10441(R) (1996); J. M. Soler, E. Artacho, J. D. Gale, A. García, J. Junquera, P. Ordejón, and D. Sánchez-Portal, J. Phys.: Condens. Matter 14, 2745 (2002).

${ }^{19}$ C. Ataca and S. Ciraci, Phys. Rev. B 82, 165402 (2010).

${ }^{20}$ H. Şahin, S. Cahangirov, M. Topsakal, E. Bekaroglu, E. Aktürk, R. T. Senger, and S. Ciraci, Phys. Rev. B 80, 155453 (2009).

${ }^{21}$ J.-A. Yan, W. Y. Ruan, and M. Y. Chou, Phys. Rev. B 77, 125401 (2008).

${ }^{22}$ S. Piscanec, M. Lazzeri, F. Mauri, A. C. Ferrari, and J. Robertson, Phys. Rev. Lett. 93, 185503 (2004).

${ }^{23}$ J. Bai, X. Zhong, S. Jiang, Y. Huang, and X. Duan, Nat. Nanotechnol. 5, 190 (2010); J. Lahiri, Y. Lin, P. Bozkurt, I. I. Oleynik, and M. Batzill, ibid. 5, 326 (2010).

${ }^{24}$ P. O. Lehtinen, A. S. Foster, A. Ayuela, A. Krasheninnikov, K. Nordlund, and R. M. Nieminen, Phys. Rev. Lett. 91, 017202 (2003).

${ }^{25}$ P. O. Lehtinen, A. S. Foster, A. Ayuela, T. T. Vehviläinen, and R. M. Nieminen, Phys. Rev. B 69, 155422 (2004).

${ }^{26}$ A. Krasheninnikov, K. Nordlund, P. O. Lehtinen, A. S. Foster, A. Ayuela, and R. M. Nieminen, Carbon 42, 1021 (2004).

${ }^{27}$ J. Friedel, Philos. Mag. 43, 153 (1952); G. F. Giuliani and G. Vignale, Quantum Theory of the Electron Liquid (Cambridge University Press, Cambridge, England, 2005).

${ }^{28}$ S. Cahangirov, M. Topsakal, and S. Ciraci, Phys. Rev. B 82, 195444 (2010). 